\title{
Fast Multipole Accelerated Finite Element - Boundary Element Analysis of Shielded Induction Heaters
}

\author{
R. V. Sabariego, P. Sergeant, J. Gyselinck, P. Dular, L. Dupré and J. Melkebeek
}

\begin{abstract}
This paper deals with the analysis of a shielded induction heater by means of a fast multipole accelerated hybrid finite element - boundary element model. It concerns an experimental setup with passive and active shielding for mitigating the stray field in the surrounding area. Numerical results of the magnetodynamic model are compared with measurements. Further, various aspects of the numerical scheme are discussed and its efficiency is evidenced.
\end{abstract}

Index Terms - fast multipole method, hybrid techniques, finite element method, boundary element method, Laplace function, induction heating

\section{INTRODUCTION}

$\mathbf{I}$ NDUCTION heating devices are used for the thermal treatment of conducting parts. This thermal treatment is achieved by means of eddy currents due to strong alternating magnetic fields. Passive and active shieldings may be employed to mitigate the magnetic field in the whole surrounding area and reduce the hazardous exposure of both the human operator and the electronic equipment. Passive shields use suitable materials to limit the losses within the shield. Active shields (coils) generate counter fields opposite to the main one to be reduced. The design of the shielding must minimize any modification of the thermal process [1]. Moreover, the accessibility of the induction heater mush be guaranteed, what strongly constrains the area in which shields can be placed. An experimental setup of an induction heater with passive and active shielding was built [1].

A hybrid finite element - boundary element (FE-BE) model is particularly suited for solving open electromagnetic field problems that comprise eddy currents [2]. The FE method easily accounts for conducting media, while the BE method provides a rigorous treatment for open problems. However, the BE part generates dense blocks in the system matrix and significantly limits the size of the problems to be handled. This limitation can be overcome by applying the fast multipole

Manuscript received June 22, 2005. The research has been carried out in the frame of the Inter-University Attraction Poles IAP P5/34 funded by the Belgian federal government.

R. V. Sabariego and P. Dular are with the Dept. of Electrical Engineering and Computer Science, Institut Montefiore, University of Liège, Sart Tilman Campus, Building B28, B-4000 Liège, Belgium (e-mail: R.Sabariego@ulg.ac.be). P. Dular is a Research Associate with the Belgian National Fund for Scientific Research (F.N.R.S.).

P. Sergeant, L. Dupré and J. Melkebeek are with the Dept. of Electrical Energy, Systems and Automation, Ghent University, Sint-Pietersnieuwstraat 41, B-9000 Ghent, Belgium.

J. Gyselinck is with the Dept. of Electrical Engineering, Université Libre de Bruxelles (ULB), Avenue Franklin Roosevelt 50, B-1050 Brussels, Belgium. method (FMM) [3], which reduces both the memory requirements and the computation time. This so-called acceleration method has already been successfully applied to models in both the high frequency [4] and the low frequency domain [5]. An adaptive truncation scheme for the 3D Laplace Green function allows to further accelerate the resolution of the system [6].

This paper deals with the application of the FMM to the hybrid analysis of a shielded induction heater. In the following section, we briefly outline both the magnetodynamic model and the FMM. The induction heater under study and practical aspects of the hybrid FE-BE model are described in Section III. Furthermore, numerical results are compared with measurements. A discussion on the computational cost is also included. Finally, we draw some conclusions in Section IV.

\section{FAST MUltipole ACCELERATEd FE-BE MOdeL}

\section{A. 3D Hybrid FE-BE model}

We consider a magnetodynamic problem in $\mathbb{R}^{3}$. The $\mathrm{FE}$ method is used in a domain $\Omega$ with boundary $\Gamma$ while the BE method takes into account the space exterior to $\Omega$. The eddy current conducting part of $\Omega$ is denoted $\Omega_{c}$ and the nonconducting one $\Omega_{c}^{C}$. Source conductors, with a given current density $\underline{j}_{s}$, define the domain $\Omega_{s} \subset \Omega_{c}^{C}$.

Adopting the magnetic field formulation, the general expression of the magnetic field $\underline{h}$ in $\Omega$ is $\underline{h}=\underline{h}_{s}+\underline{h}_{r}-\operatorname{grad} \phi$, with $\underline{h}_{s}$ a source field satisfying $\operatorname{curl} \underline{h}_{s}=\underline{j}_{s}, \underline{h}_{r}$ the reaction field in $\Omega_{c}$, and $\phi$ the reaction magnetic scalar potential in $\Omega_{c}^{C}$.

The magnetodynamic $\underline{h}-\phi$ formulation is obtained from the weak form of the Faraday equation:

$$
\begin{aligned}
& \partial_{t}\left(\mu \underline{h}, \underline{h^{\prime}}\right)_{\Omega}+\left(\sigma^{-1} \operatorname{curl} \underline{h}, \operatorname{curl} \underline{h}^{\prime}\right)_{\Omega_{c}}+ \\
& \left(\sigma^{-1} \underline{j}_{s}, \operatorname{curl} \underline{h^{\prime}}\right)_{\Omega_{s}}+\partial_{t}\left\langle\underline{\underline{n}} \cdot \underline{b}, \phi^{\prime}\right\rangle_{\Gamma}=0, \quad \forall \underline{h^{\prime}} \in F_{h \phi}(\Omega),
\end{aligned}
$$

where $\mu$ is the magnetic permeability, $\sigma$ is the electric conductivity; $\underline{n}$ is the unit normal vector on $\Gamma$ pointing into $\Omega$; $(\cdot, \cdot)_{\Omega}$ and $\langle\cdot, \cdot\rangle_{\Gamma}$ denote a volume integral in $\Omega$ and a surface integral on $\Gamma$ of the product of their arguments; $F_{h \phi}(\Omega)$ is the function space defined on $\Omega$ and containing the basis functions for $\underline{h}$ (coupled to $\phi$ ) as well as for the test function $\underline{h}^{\prime}$ [7].

The coupling with the BE model is done through the surface integral in (1), which can be expressed as

$$
\partial_{t}\left\langle\underline{n} \cdot \underline{b}, \phi^{\prime}\right\rangle_{\Gamma}=-\partial_{t}\left\langle\mu \underline{n} \cdot \operatorname{grad} \phi(\underline{r}), \phi^{\prime}\right\rangle_{\Gamma}+\partial_{t}\left\langle\mu \underline{n} \cdot \underline{h}_{s}, \phi^{\prime}\right\rangle_{\Gamma} .
$$


The scalar potential and its normal derivative in a point on $\Gamma$ are given by

$$
\begin{aligned}
\phi(\underline{r}) & =\mu^{-1} \int_{\Gamma} q G \mathrm{~d} \Gamma, \\
\underline{n} \cdot \operatorname{grad} \phi(\underline{r}) & =\frac{1}{2} q+\mu^{-1} \int_{\Gamma} \underline{n} \cdot \operatorname{grad} G q \mathrm{~d} \Gamma,
\end{aligned}
$$

where $q$ is an equivalent magnetic charge on $\Gamma$ and $G=1 / 4 \pi \rho$ is the 3D Laplace Green function, $\rho$ being the distance between a source point $\underline{r}_{s}$ (on $\Gamma$ ) and an observation point $\underline{r}_{o}$ (in $\mathbb{R}^{3} \backslash \Omega$ ).

The weak form of (3) reads:

$$
\left\langle\phi, q^{\prime}\right\rangle_{\Gamma}=\left\langle\mu^{-1} \int_{\Gamma} q G \mathrm{~d} \Gamma, q^{\prime}\right\rangle_{\Gamma}, \quad \forall q^{\prime} \in F_{q}(\Gamma),
$$

where $F_{q}(\Gamma)$ is the function space defined on $\Gamma$ which contains the basis functions for $q$ and the test function $q^{\prime}$.

Applying the Galerkin method to (1) and (5), the system of equations of the hybrid model is obtained. Edge basis functions are employed for $\underline{h}$, resulting in sparse blocks in the system matrix. The terms involving $\phi$ produce dense blocks.

\section{B. Fast multipole method}

The fast multipole method decomposes the boundary $\Gamma$ into groups of elements [3]. Let $\Gamma_{s}$ be a source group with center $\underline{r}_{s c}$ and a source point $\underline{r}_{s}$, and $\Gamma_{o}$ an observation group with center $\underline{r}_{o c}$ and an observation point $\underline{r}_{o}$. We define the vectors $\underline{r}=\underline{r}_{o}-\underline{r}_{o c}=(r, \theta, \phi), \underline{r}_{c}=\underline{r}_{o c}-\underline{r}_{s c}=\left(r_{c}, \theta_{c}, \phi_{c}\right)$ and $\underline{r}^{\prime}=\underline{r}_{s c}-\underline{r}_{s}=\left(r^{\prime}, \theta^{\prime}, \phi^{\prime}\right)$ in spherical coordinates. The interactions between distant groups are determined by means of the multipole expansion of $G$, i.e.

$$
\begin{gathered}
G=\Re\left(\sum_{m=0}^{\infty} \sum_{n=-m}^{m} \sum_{u=0}^{\infty} \sum_{v=-u}^{u} \mathcal{D}_{m, n} \mathcal{T}_{m+u, n+v} \mathcal{A}_{u, v}\right), \\
\mathcal{D}_{m, n}=\frac{r^{m} \mathcal{L}_{m}^{n}(\theta, \phi)}{(m+n) !} \\
\mathcal{T}_{m+u, n+v}=\frac{(m+u-(n+v)) !}{4 \pi r_{c}^{m+u+1}} \mathcal{L}_{m+u}^{n+v}\left(\theta_{c},-\phi_{c}\right), \\
\mathcal{A}_{u, v}=\frac{r^{\prime u} \mathcal{L}_{u}^{v}\left(\theta^{\prime}, \phi^{\prime}\right)}{(u+v) !}
\end{gathered}
$$

where $\mathcal{L}_{m}^{n}(\theta, \phi)=P_{m}^{n}(\cos \theta) e^{-\imath n \phi}, P_{m}^{n}$ being the Legendre function of degree $m$ and order $n$. The imaginary number is denoted $\imath$ and $\Re$ indicates the real part.

In practice, the multipole expansion (6) must be truncated by considering $0 \leq m, u \leq p$, where $p$ must be sufficiently large to restrain the error to a value $\varepsilon$. We adopt an adaptive truncation scheme which has been proved to be more economic than the conventional choice [6].

The expansion of $\operatorname{grad} G$ is required as well. It is straightforwardly obtained by deriving (7) with respect to the coordinates of the observation point.

Let us consider the radii of the source and observation groups, $R_{s}=\max _{\Gamma_{s}}\left(r^{\prime}\right), R_{o}=\max _{\Gamma_{o}}(r)$, and the distance between their centers $d=r_{c}$. Two groups $\Gamma_{s}$ and $\Gamma_{o}$ are said to be 'far' groups if $R_{s} / d<\tau$ and $R_{o} / d<\tau$, where $d$ is the distance between the group centers and where $\tau$ is chosen smaller than $1 / 2$. As a rule of thumb, the optimum value of $\tau$ lies in the interval $[1 / 4,1 / 5]$, i.e., an observation group $\Gamma_{o}$ is far from a source group $\Gamma_{s}$ if it is outside the sphere of radius $R_{f a r} \in\left[3 R_{s}, 4 R_{s}\right]$ with origin the center $\underline{r}_{s c}$ of $\Gamma_{s}$.

\section{ANALYSis OF THE INDUCTION HEATER}

\section{A. Description of the problem}

The two-dimensional layout (axisymmetric and symmetric with regard to the $z=0$ plane) of the induction heater is depicted in Fig. 1. It comprises an excitation coil carrying a sinusoidal current (40 A at $1 \mathrm{kHz}$ ), a passive steel shield ( $190 \mathrm{~mm}$ high, $0.65 \mathrm{~mm}$ wide, $\sigma=5.910^{6} \mathrm{~S} / \mathrm{m}, \mu_{r}=372$ ) and an active shield constituted by 18 compensation coils ( 9 above and 9 below the symmetry plane, optimal current $\left.0.1162 e^{-49.3^{\circ}{ }^{\imath}} \mathrm{mA}, \mu_{r}=1\right)$. The workpiece is a cylindrical aluminium plate $($ radius $=191 \mathrm{~mm}$, height $=10 \mathrm{~mm}, \sigma=$ $3.710^{7} \mathrm{~S} / \mathrm{m}, \mu_{r}=1$ ). Further details about the geometry can be found in [1].

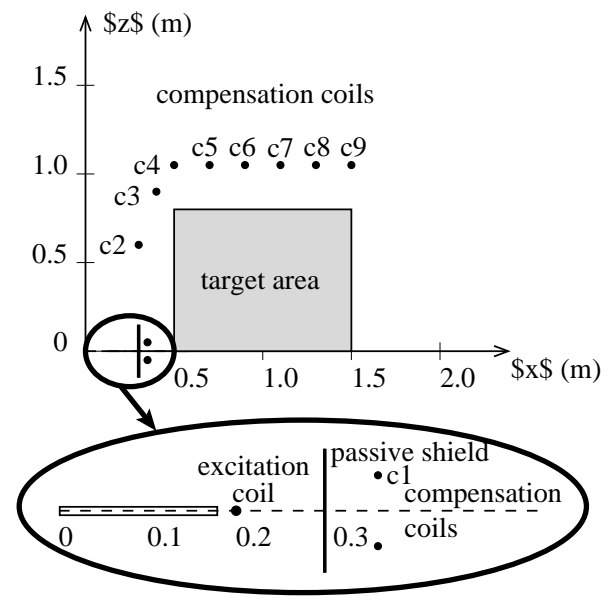

Fig. 1. 2D axisymmetrical layout of the induction heater and shields

The magnetic field in the target area (see Fig. 1), the power dissipation in both the active and passive shields and the influence of the shields in the heating process have been reduced by using a genetic algorithm (GA) [1]. The GA returns the position and height of the passive shield, the optimal currents for the active shield, and the number of turns of the coils. The optimal coordinates and the number of turns of the nine coils above the symmetry plane $(z=0)$ for the optimization at $1 \mathrm{kHz}$ can be found in Table I.

In order to emphasize the capabilities of the FMM, we consider a full three-dimensional model. Note that the problem is then multiply connected and a cut must be defined to ensure the unicity of the potential [8].

The linear eddy current problem is solved by applying the complex formalism. The source magnetic field $\underline{h}_{s}$ due to the excitation and the compensation coils is calculated by means of the Biot-Savart law. To this end, the excitation coil and the compensation coils are discretized in 88 hexahedra and 60 line segments each, respectively.

The FE domain $\Omega$ can be thus restricted to the workpiece and the passive shield $\Omega_{c}=\Omega_{w} \cup \Omega_{p}$. We adopt edge basis functions for $\underline{h}$ and piecewise linear basis functions for $q$. 
TABLE I

OPTIMAL COIL POSITIONS AND NUMBER OF TURNS

\begin{tabular}{c||c|c|c}
\hline Coil & $x(\mathrm{~m})$ & $z(\mathrm{~m})$ & Turns \\
\hline \hline c1 & 0.365 & 0.08 & -1 \\
c2 & 0.300 & 0.60 & 8 \\
c3 & 0.400 & 0.90 & 4 \\
c4 & 0.500 & 1.15 & -4 \\
c5 & 0.700 & 1.15 & 6 \\
c6 & 0.900 & 1.15 & -4 \\
c7 & 1.100 & 1.15 & 4 \\
c8 & 1.300 & 1.15 & -3 \\
c9 & 1.500 & 1.15 & 2 \\
\hline
\end{tabular}

Different levels of mesh refinement are considered. We pay special attention to the discretization of the workpiece. When the FMM is applied, the surface of the workpiece $\Gamma_{w}$ is split up into $25,36,45$ or 60 groups. The CPU time and memory requirements for these different grouping schemes are shown in Tables II and III. Note that for a particular mesh there is an optimal group distribution: employing more groups yields a higher computation time for solving the system of equations and more storage cost for the FMM data structures, while using less groups increases the assembly time and the memory requirements for the BE part. The optimal number of groups increases with the number of unknowns.

TABLE II

CPU TIME (MIN) FOR DIFFERENT GROUPING SCHEMES

\begin{tabular}{c||c||c||c||c}
\hline$N_{B E}$ & $\# g=25$ & $\# g=36$ & $\# g=45$ & $\# g=60$ \\
\hline \hline 584 & 1.7 & 2.2 & 2.3 & 2.3 \\
1096 & 4.7 & 4.7 & 4.7 & 4.4 \\
1152 & 6.4 & 5.5 & 6.2 & 6.1 \\
1476 & 9.3 & 8.2 & 8.2 & 8.6 \\
1912 & 14.2 & 14.6 & 15.7 & 15.4 \\
2776 & 32.2 & 28.8 & 29.2 & 27.5 \\
\hline
\end{tabular}

TABLE III

MEMORY (MB) REQUIREMENTS FOR DIFFERENT GROUPING SCHEMES

\begin{tabular}{c||c||c||c||c}
\hline$N_{B E}$ & $\# g=25$ & $\# g=36$ & $\# g=45$ & $\# g=60$ \\
\hline \hline 584 & 20.8 & 22.1 & 22.6 & 23.2 \\
1096 & 33.3 & 33.4 & 34 & 37.4 \\
1152 & 38.4 & 35.4 & 40.2 & 43.5 \\
1476 & 47.5 & 47.4 & 49.5 & 46.3 \\
1912 & 75.6 & 73.9 & 73.9 & 73.1 \\
2776 & 118.1 & 114.7 & 114.6 & 111.9 \\
\hline
\end{tabular}

An analogous analysis could be done for the surface of the passive shield $\Gamma_{p}$.

\section{B. Calculation results}

The hybrid FE-BE discretization yields 41706 unknowns: 36618 for the FE part and 5088 for the BE part (see Fig. 2). This is the finest mesh in Tables II, III and IV. The optimal group distribution is found to be 60 groups for the workpiece $\Gamma_{w}$ and 160 groups for the passive shield $\Gamma_{p}$. The maximum and average truncation number are $p_{\max }=10$ and $p_{a v}=3$ for $R_{f a r}=0.086$ and $\epsilon=10^{-6}$.

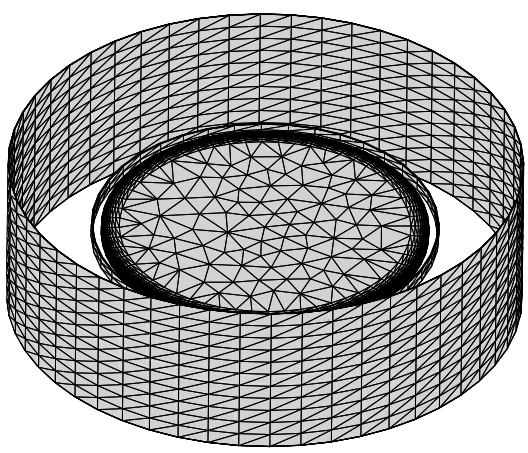

Fig. 2. 3D mesh of the induction heater with only the passive shield

The flux pattern is represented in Fig. 3. Herein, the shielding effect is evidenced.

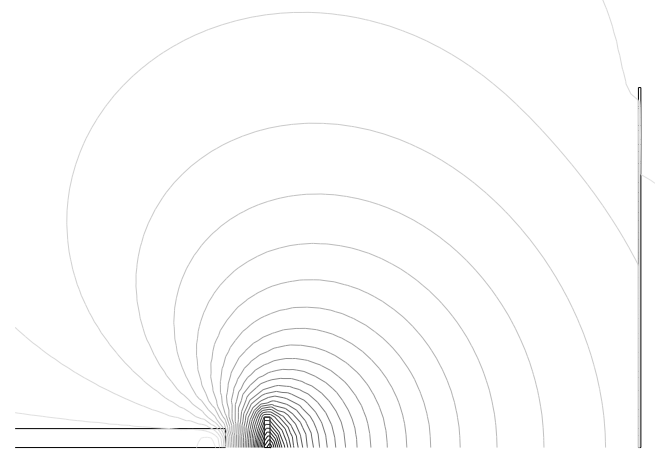

Fig. 3. Flux lines with active and passive shielding

The normal component of the magnetic flux density $\underline{b}$ in the plate in the interval [radius $-5 \delta$, radius] along the $x$-axis, with $\delta$ the skin depth, is shown in Fig. 4. An excellent agreement between results obtained by an axisymmetrical 2D FE model and the 3D hybrid FE-BE model is observed.

Simulations results are then compared with measurements performed on the experimental setup. The modulus of the magnetic flux density $\underline{b}$ measured along the $x$-axis in the target area (see Fig.1) is compared with the one obtained by means of the FMM accelerated FE-BE model in Fig. 5 for two different cases: with only the passive shield and with both passive and active shields. The case without shields is shown as a reference. Note that in the case with both passive and active shields, the low field levels in the target area are highly sensitive to the perfect supply of the compensation coils (amplitude and phase), their precise position and other factors linked to the set-up itself (e.g. steel bars in the ceiling and the floor) that influence the measurements. The numerical model shows sufficient correlation with the measurements and provides a good representation of the device.

\section{Computational cost}

The system of algebraic equations is solved by means of the iterative solver GMRES [9] with ILU-preconditioning on a 3.2 GHz Mobile Intel Pentium 4 Processor. In case of FMM 


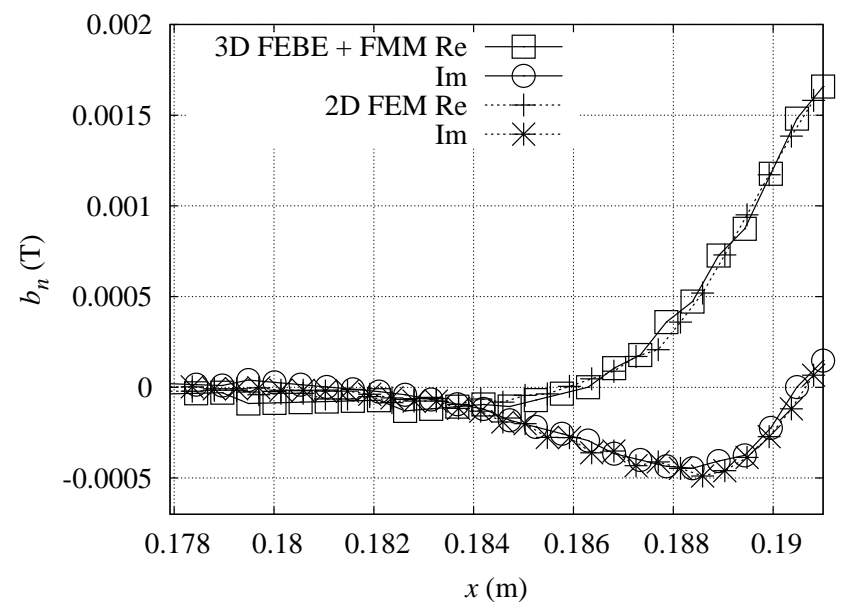

Fig. 4. Real and Imaginary of the normal component of the magnetic flux density (T) in the workpiece. Comparison between results obtained by a 2D axisymmetric FE model and a 3D FE-BE model

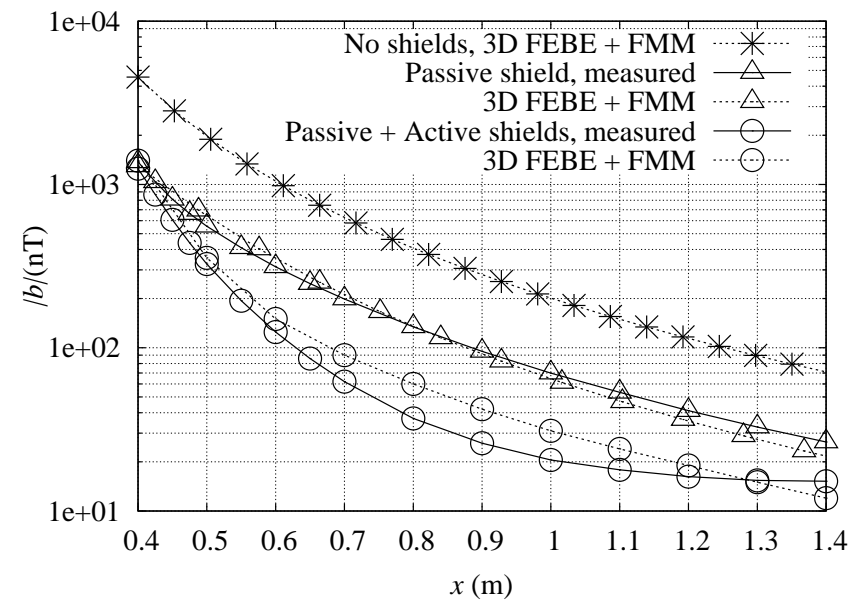

Fig. 5. Modulus of the magnetic flux density (nT) in the target area. Comparison of simulated and experimental results

acceleration, the preconditioning is based on the sparse matrix comprising the complete FE contribution but only the BE nearfield interactions.

The CPU time and the memory requirements for the different discretizations of the workpiece (with $\Gamma_{w}$ split up into the corresponding optimal group distribution) are shown in Table IV. It illustrates the efficiency of the FMM as the number of BE unknowns increases. With the last mesh, e.g., the accelerated FE-BE analysis is roughly 24 times faster than the non-accelerated one and the savings in memory reach $58 \%$.

The solution of the complete system (workpiece, passive and active shielding) was obtained after $\approx 72 \mathrm{~h}$ and $0.83 \mathrm{~h}$ for the non-accelerated and the accelerated model respectively. With regard to the memory requirements, the FMM acceleration reduces them from $310 \mathrm{MB}$ to $87 \mathrm{MB}$.

\section{CONCLUSION}

An induction heater with a passive and an active shielding has been studied. The resolution of this $3 \mathrm{D}$ eddy current
TABLE IV

CPU TIME (MIN) AND MEMORY (MB) REQUIREMENTS FOR DIFFERENT MESHES

\begin{tabular}{c|c||c|c||c|c||c|c}
\hline \multicolumn{2}{c||}{ Unknowns } & \multicolumn{2}{c||}{ FE-BE } & \multicolumn{2}{c||}{ FE-BE + FMM } & \multicolumn{2}{c}{ Factor } \\
\hline FE & BE & CPU & mem & CPU & mem & CPU & mem \\
\hline \hline 3238 & 584 & 4.9 & 21.8 & 1.7 & 20.8 & 2.8 & 1 \\
6350 & 1096 & 28.7 & 55.2 & 4.7 & 33.3 & 6 & 1.6 \\
9754 & 1152 & 34.8 & 57.0 & 5.5 & 35.4 & 6.3 & 1.6 \\
12746 & 1476 & 74.2 & 89.4 & 8.2 & 47.4 & 9 & 1.9 \\
21774 & 1912 & 166.3 & 163.9 & 14.2 & 73.8 & 11.7 & 2.2 \\
32550 & 2776 & 649.8 & 265.5 & 27.5 & 111.9 & 23.6 & 2.4 \\
\hline
\end{tabular}

problem by means of the FMM accelerated hybrid FE-BE model has been elaborated.

In order to evidence the capabilities of the FMM, a full 3D model has been considered. The accuracy of the model has been validated by comparing the results to those obtained by an axisymmetrical 2D FE model. Furthermore, a good agreement between calculated and experimental results has been observed. The numerical model provides a good representation of the device.

The efficiency of the FMM has been illustrated by comparing the results obtained for different discretizations. Significant savings in computation time and storage requirements are achieved. We have observed an increase of the number of iterations when applying the FMM. This is mainly due to the fact that the preconditioning disregards the far field interactions. This raise is largely outweighed by the fact the aggregation, translation and disaggregation matrices are never evaluated explicitly.

\section{REFERENCES}

[1] P. Sergeant, U. Adriano, L. Dupré, O. Bottauscio, M. D. Wulf, M. Zucca, and J. Melkebeek, "Passive and active electromagnetic shielding of induction heaters," IEEE Transactions on Magnetics, vol. 40, no. 2, pp. 675-678, March 2004.

[2] J. Fetzer, S. Kurz, G. Lehner, and W. M. Rucker, "Analysis of an actuator with eddy currents and iron saturation: Comparison between a fem and a bem-fem coupling approach," IEEE Transactions on Magnetics, vol. 35, no. 3, pp. 1793-1796, May 1999.

[3] V. Rokhlin, "Rapid solution of integral equations of classical potential theory," Journal of Computational Physics, vol. 60, no. 2, pp. 187-207, 1985.

[4] J. M. Song and W. C. Chew, "Multilevel fast-multipole algorithm for solving combined field integral equations of electromagnetic scattering," Microwave and Optical Technology Letters, vol. 10, no. 1, pp. 14-19, September 1995.

[5] A. Buchau, C. J. Huber, W. Rieger, and W. M. Rucker, "Fast bem computations with the adaptive multilevel fast multipole method," IEEE Transactions on Magnetics, vol. 36, no. 4, pp. 680-684, July 2000.

[6] R. V. Sabariego, J. Gyselinck, P. Dular, C. Geuzaine, and W. Legros, "Fast multipole acceleration of the hybrid finite element-boundary element analysis of 3d eddy current problems," IEEE Transactions on Magnetics, vol. 40, no. 2, pp. 1278-1281, March 2004.

[7] P. Dular, C. Geuzaine, and W. Legros, "A natural method for coupling magnetodynamic h-formulations and circuit equations," IEEE Transactions on Magnetics, vol. 35, no. 3, pp. 1626-1629, May 1999.

[8] J. C. Vérité, "Calculation of multivalued potentials in exterior regions," IEEE Transactions on Magnetics, vol. 23, no. 3, pp. 1881-1887, May 1987.

[9] Y. Saad and M. H. Schultz, "GMRES: A generalized minimal residual algorithm for solving nonsymmetric linear systems," SIAM Journal of Scientific and Statistical Computing, vol. 7, no. 3, pp. 856-869, July 1986. 